\title{
Stabilisation des phases monocristallines quadratique et cubique dans $\mathrm{BaTiO}_{3}$
}

\author{
J. Amami ${ }^{1}$, C. Goutaudier ${ }^{1}$, M. Trabelsi-Ayadi ${ }^{2}$ et G. Boulon ${ }^{1}$ \\ ${ }^{1}$ Laboratoire de Physico-Chimie des Matériaux Luminescents, Université Claude Bernard \\ Lyon 1, UMR CNRS 5620, 10 rue Ampère, Bât. A. Kastler, 69622 Cedex, Villeurbanne, \\ France \\ ${ }^{2}$ Laboratoire de Physico-Chimie Minérale, Faculté des Sciences de Bizerte, 7021 Zarzouna, \\ Tunisie
}

\begin{abstract}
Single crystals of $\mathrm{Ba}_{1-\mathrm{x}} \mathrm{Yb}_{\mathrm{x}} \mathrm{TiO}_{3}$ and $\mathrm{Ba}_{1-\mathrm{x}} \mathrm{Sr}_{\mathrm{x}} \mathrm{TiO}_{3}$ with tetragonal structure were grown by the floating zone (FZ) method using an LHPG (Laser Heated Pedestal Growth) technique. The grown crystals, typically $1 \mathrm{~mm}$ in diameter and $8 \mathrm{~mm}$ in length, were yellowish transparent. Substitution of more than 2.5 at \% $\mathrm{Sr}$ for $\mathrm{Ba}$ in $\mathrm{BaTiO}_{3}$ and addition of 3 to 5 at \% $\mathrm{Ln}(\mathrm{Ln}=\mathrm{Yb}, \mathrm{Eu})$, were effective in preventing the formation of the hexagonal phase. The $\mathrm{Eu}^{3+}$ emission spectra recorded at room temperature demonstrate a significant intensity enhancement of the ${ }^{5} \mathrm{D}_{0} \rightarrow{ }^{7} \mathrm{~F}_{1}$ magnetic dipolar transition compared to the ${ }^{5} \mathrm{D}_{0} \rightarrow{ }^{7} \mathrm{~F}_{2}$ electric dipolar transition.
\end{abstract}

Résumé. Les fibres monocristallines de $\mathrm{Ba}_{1-\mathrm{x}} \mathrm{Yb}_{\mathrm{x}} \mathrm{TiO}_{3}$ et $\mathrm{Ba}_{1-\mathrm{x}} \mathrm{Sr}_{\mathrm{x}} \mathrm{TiO}_{3}$ de structure quadratique ont été préparées par la méthode de la zone flottante (FZ) en utilisant la technique LHPG (Laser Heated Pedestal Growth). Les échantillons élaborés, de dimensions $1 \mathrm{~mm} \times 8 \mathrm{~mm}$, sont jaunâtres et transparentes. La substitution du baryum par plus de $2,5 \mathrm{~mol} \%$ de $\mathrm{Sr}$ dans $\mathrm{BaTiO}_{3}$ et le dopage de 3 à $5 \mathrm{~mol} \%$ de $\operatorname{Ln}(\operatorname{Ln}=\mathrm{Yb}, \mathrm{Eu})$ évitent la formation de la phase hexagonale. Le spectre d'émission de $\mathrm{Eu}^{3+}$ enregistré à température ambiante montre le changement de structure traduit par la forte intensité de la transition dipolaire magnétique ${ }^{5} \mathrm{D}_{0} \rightarrow{ }^{7} \mathrm{~F}_{1}$ comparée à celle de la transition dipolaire électrique ${ }^{5} \mathrm{D}_{0} \rightarrow{ }^{7} \mathrm{~F}_{2}$.

\section{INTRODUCTION}

Le titanate de baryum $\mathrm{BaTiO}_{3}$, reconnu pour ses propriétés électriques, présente 5 structures polymorphes. Au dessus de la température ambiante, les transitions structurales quadratique $\longrightarrow$ cubique $\longrightarrow$ hexagonale sont successivement observées vers $120^{\circ} \mathrm{C}$ et $1460{ }^{\circ} \mathrm{C}[1]$.

La croissance cristalline à partir d'un bain fondu stæchiométrique conduit à des cristaux de structure hexagonale, cette phase pouvant être maintenue dans un état métastable jusqu'à la température ambiante. La phase quadratique, ferroélectrique, est actuellement très recherchée en raison d'applications potentielles en optique non linéaire [2-4] et l'obtention de tels monocristaux ne peut être réalisée qu'à partir de techniques de flux. 
Ce travail propose une première tentative de stabilisation de la phase quadratique par addition d'un troisième constituant formant une solution solide avec $\mathrm{BaTiO}_{3}$, l'objectif ultime étant d'élaborer directement des monocristaux de structure quadratique par la technique de zone flottante LHPG et s'affranchir de l'utilisation d'un flux. Nous avons focalisé nos travaux sur l'étude des conditions d'obtention de cette phase à partir des systèmes $\mathrm{BaTiO}_{3}-\mathrm{SrTiO}_{3}$ et $\mathrm{BaTiO}_{3}-\mathrm{Ln}_{2} \mathrm{O}_{3}$ sous forme de fibres monocristallines.

\section{CONDITIONS EXPÉRIMENTALES}

Les différentes phases étudiées ont été synthétisées d'abord sous forme de poudre par réaction à l'état solide. $\mathrm{BaTiO}_{3}$ est préparé à partir d'un mélange stochiométrique de $\mathrm{BaCO}_{3}$ et $\mathrm{TiO}_{2}$ auquel sont ajoutées diverses proportions de $\mathrm{Ln}_{2} \mathrm{O}_{3}$ ou $\mathrm{Sr}\left(\mathrm{NO}_{3}\right)_{2}$, la pureté des oxydes sélectionnés étant de 99,99\%. L'homogénéisation des réactifs est assurée par broyage et agitation mécanique. Le mélange est ensuite compacté sous forme d'une pastille pressée sous quatre tonnes.

L'échantillon est soumis à deux traitements thermiques successifs à haute température $\left(1000^{\circ} \mathrm{C}\right)$ pendant 16 heures, dans le but d'initier la réaction de formation des phases et de fritter la pastille. Après densification des pastilles céramiques, des barreaux fins de dimensions typiques : $1 \times 1 \times 2,5 \mathrm{~mm}^{3}$ sont découpées. Les fibres cristallines sont ensuite élaborées sous air par la technique LHPG (Laser Heated Pedestal Growth) à partir des barreaux céramiques décrits ci-dessus avec des vitesses de tirage comprises entre 10 et $15 \mathrm{~mm} / \mathrm{h}$. Les parties transparentes des fibres ont été ensuite caractérisées par diffraction des rayons X.

\section{RESULTATS ET DISCUSSION}

Le système binaire $\mathrm{BaTiO}_{3}-\mathrm{SrTiO}_{3}$ [5-7] réalisé sur poudre présente une solution solide cubique étendue au maximum jusqu' à $23 \mathrm{~mol} \% \mathrm{Sr}$. Elle fond de façon congruente de 1,5 à 2,5 mol \% Sr selon les auteurs, pour une température proche de $1580{ }^{\circ} \mathrm{C}$. La solution solide cubique se transformerait en quadratique entre 120 et $60^{\circ} \mathrm{C}[8]$.

La figure 1 montre les diffractogrammes de RX enregistrés à partir de deux fibres broyées, qui étaient parfaitement transparentes et exemptes d'inclusions.

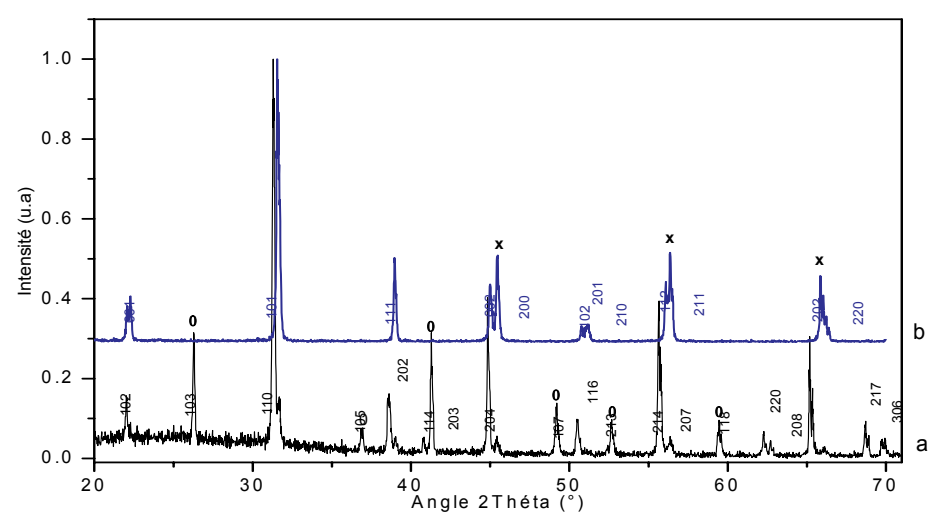

Figure 1. Diffractogrammes de rayons $\mathrm{X}$ de $\mathrm{BaTiO}_{3}$ (a) et $\mathrm{Ba}_{0,975} \mathrm{Sr}_{0,025} \mathrm{TiO}_{3}$ (b) à température ambiante (raies caractéristiques de la structure quadratique $(\mathbf{x})$ et hexagonale $(\mathbf{0}))$. 
La première, $\mathrm{BaTiO}_{3}$ pur, présente la structure haute température hexagonale (groupe d'espace $\mathrm{P}_{3} / \mathrm{mmc}$ ) conformément à la fiche JCPDS $\mathrm{n}^{\circ}$ 34-0129. La seconde fibre, de composition nominale $\mathrm{Ba}_{0,975} \mathrm{Sr}_{0,025} \mathrm{TiO}_{3}$, présente un diffractogramme complètement différent qui ne peut être indexé dans la structure hexagonale. Les structures cubique et quadratique de $\mathrm{BaTiO}_{3}$ (fiches JCPDS 31-0174 et 05-0626) étant très proches, il est délicat de les différencier par leur diffractogramme. Les mesures DRX ont donc été reprises à température ambiante puis à $135^{\circ} \mathrm{C}$, c'est-à-dire de part et d'autre de la transition structurale, avec des temps de comptage plus longs. Dans le diffractogramme basse température, le dédoublement de raies correspondant aux indices hkl 200, 211 et 220 permet d'affirmer sans ambiguïté que la fibre contenant $2,5 \%$ de Strontium a la structure quadratique (P4mm) et est parfaitement monophasée.

La figure 2 donne une représentation du système $\mathrm{BaO}-\mathrm{TiO}_{2}-\mathrm{Ln}_{2} \mathrm{O}_{3}$ qui n'est pas connu mais trois composés définis ternaires sont répertoriés avec pratiquement toutes les terres rares : $\mathrm{Ba}_{12} \mathrm{Ln}_{4,67} \mathrm{Ti}_{8} \mathrm{O}_{35}$, $\mathrm{BaLn}_{2} \mathrm{Ti}_{4} \mathrm{O}_{12}$ et $\mathrm{BaLn}_{2} \mathrm{Ti}_{3} \mathrm{O}_{10}$; ils n'appartiennent toutefois pas à la coupe $\mathrm{BaTiO}_{3}-\mathrm{Ln}_{2} \mathrm{O}_{3}$.

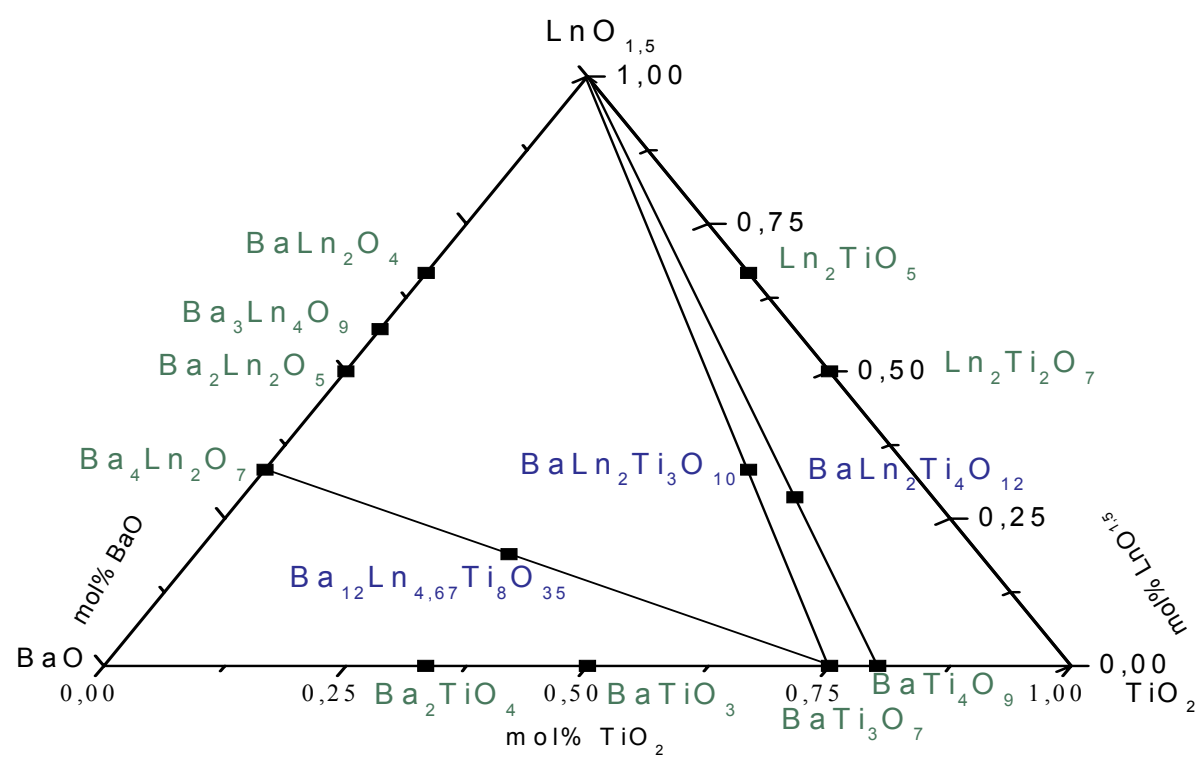

Figure 2. Phases solides connues dans le système ternaire $\mathrm{BaO}-\mathrm{TiO}_{2}-\mathrm{LnO}_{1.5}$.

Dans un premier temps, la terre rare choisie pour étudier son influence sur la structure de $\mathrm{BaTiO}_{3}$ a été l'ytterbium parce qu'il n'absorbe pas dans le visible. Des quantités allant de 0,1 à 8 mol\% d'ytterbium ont été ajoutées puis les fibres cristallines ont été tirées. Tous les cristaux, de $1 \mathrm{~mm}$ de diamètre et 8 $\mathrm{mm}$ de longueur, sont transparents et teintés en jaune (Fig. 3), mais cette coloration s'estompe après un recuit sous oxygène. La figure 4 présente les diffractogrammes enregistrés à température ambiante sur les fibres broyées. Pour les très faibles quantités d'ytterbium (inférieure à 0,5\%), la fibre est de structure hexagonale. Entre 0,5 et $3 \mathrm{~mol} \% \mathrm{Yb}$, la fibre obtenue est un mélange de phase hexagonale et quadratique, et ce n'est qu'entre 3 et $5 \mathrm{~mol} \%$ Yb ajouté que la structure monocristalline de $\mathrm{BaTiO}_{3}$ est uniquement quadratique. Au delà de cette concentration une phase secondaire apparaît, dont la nature n'a pas encore été identifiée. Elle ne correspond pas aux phases ternaires répertoriées à ce jour dans le système $\mathrm{BaO}-\mathrm{TiO}_{2}-\mathrm{LnO}_{1.5}$. 


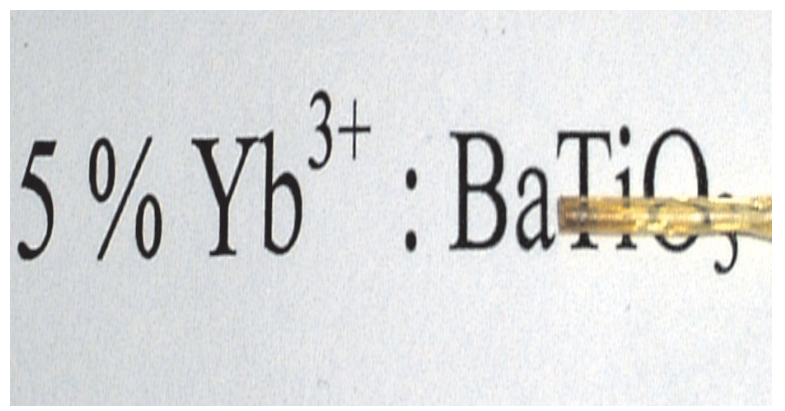

Figure 3. Exemple d'une fibre élaborée par LHPG.

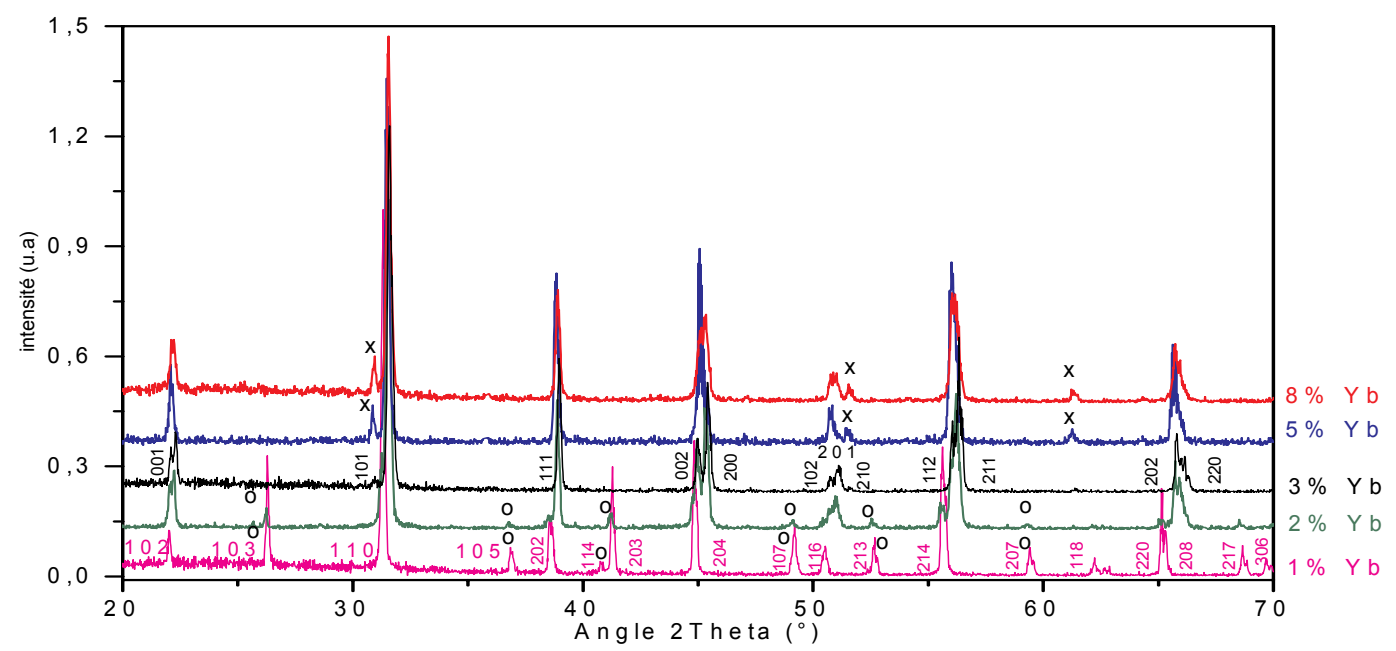

Figure 4. Diffractogrammes à température ambiante de fibres $\mathrm{BaTiO}_{3}$ contenant des quantités croissantes de $\mathrm{Yb}_{2} \mathrm{O}_{3}$ (x : phase secondaire non identifiée).

Dans un second temps, l'ion $\mathrm{Eu}^{3+}$ a été introduit en faible quantité comme sonde structurale des phases susceptibles d'être observées dans les monocristaux massifs de $\mathrm{BaTiO}_{3}$. Une étude préalable a été reportée sur les propriétés d'émission de nanocristaux dopés $\mathrm{Eu}^{3+}$ élaborés par la voie sol-gel dont les dimensions de grains étaient comprises entre 32 et $38 \mathrm{~nm}$ [9]. A la température ambiante, les phases cubique et quadratique ont été détectées selon la nature du traitement thermique des échantillons analysés. La phase quadratique a pu être stabilisée avec l'augmentation de la teneur en $\mathrm{Eu}^{3+}$ ainsi qu'avec l'augmentation de la taille des grains. Sur la figure 5 sont comparés les spectres d'émission de l'ion $\mathrm{Eu}^{3+}$ dans les fibres monocristallines des phases cubique à $423 \mathrm{~K}$ (a) et quadratique à température ambiante (b) avec un échantillon nanocristallin de structure rhomboédrique à $77 \mathrm{~K}$ (c). Le nombre et la largeur des raies d'émission ${ }^{5} \mathrm{D}_{0} \rightarrow{ }^{7} \mathrm{~F}_{0}$ de nature dipolaire-électrique forcée renseigne sur le nombre et la distribution des sites $\mathrm{Eu}^{3+}$ en substitution des cations $\mathrm{Ba}^{2+}$. La comparaison des intensités des transitions ${ }^{5} \mathrm{D}_{0} \rightarrow{ }^{7} \mathrm{~F}_{1}$, de nature dipolaire magnétique, et des transitions ${ }^{5} \mathrm{D}_{0} \rightarrow{ }^{7} \mathrm{~F}_{2}$ de nature dipolaire électrique permet, en outre, de reconnaître la présence éventuelle d'un centre d'inversion pour lequel l'intensité de ${ }^{5} \mathrm{D}_{0} \rightarrow{ }^{7} \mathrm{~F}_{1}$ est alors prédominante par rapport à ${ }^{5} \mathrm{D}_{0} \rightarrow{ }^{7} \mathrm{~F}_{2}$. Enfin, le nombre de composantes Stark de ${ }^{5} \mathrm{D}_{0} \rightarrow{ }^{7} \mathrm{~F}_{1}$, de 1 à 3 au maximum, peut aussi donner des informations sur le nombre et la symétrie ponctuelle des sites occupés. 
A l'évidence, ces spectres traduisent la diversité des phases cristallines. Il n'y a pratiquement pas de corrélation entre les positions des composantes Stark des transitions ${ }^{5} \mathrm{D}_{0} \rightarrow{ }^{7} \mathrm{~F}_{1}$ et ${ }^{5} \mathrm{D}_{0} \rightarrow{ }^{7} \mathrm{~F}_{2}$ dans les trois échantillons. Les transitions dipolaires magnétiques ${ }^{5} \mathrm{D}_{0} \rightarrow{ }^{7} \mathrm{~F}_{1}$ sont beaucoup plus intenses que les transitions dipolaires électriques ${ }^{5} \mathrm{D}_{0} \rightarrow{ }^{7} \mathrm{~F}_{2}$ dans la phase cristalline quadratique de la fibre (b) indiquant la présence d'un centre d'inversion dans cette phase alors que ce n'est pas le cas pour les deux autres phases. La phase cubique (a) comporte 4 composantes ${ }^{5} \mathrm{D}_{0} \rightarrow{ }^{7} \mathrm{~F}_{1}$ en relation avec probablement l'existence de deux types de sites qui ne sont pas détectés par les deux raies alors attendues pour la transition ${ }^{5} \mathrm{D}_{0} \rightarrow{ }^{7} \mathrm{~F}_{0}$. Par contre la phase quadratique (b) montre une raie ${ }^{5} \mathrm{D}_{0} \rightarrow{ }^{7} \mathrm{~F}_{0}$ de forte intensité associée à deux autres raies d'intensité beaucoup plus faible et qui pourrait traduire une pluralité de sites en raison des effets de compensation de charge lors de la substitution de $\mathrm{Ba}^{2+}$ par $\mathrm{Eu}^{3+}$. Enfin, la phase nanocristalline contient une distribution continue de sites au vu de l'absence de transitions structurées pour les trois domaines spectraux relatifs à ${ }^{5} \mathrm{D}_{0} \rightarrow{ }^{7} \mathrm{~F}_{0},{ }^{5} \mathrm{D}_{0} \rightarrow{ }^{7} \mathrm{~F}_{1}$ et ${ }^{5} \mathrm{D}_{0} \rightarrow{ }^{7} \mathrm{~F}_{2}$.

\section{CONCLUSION}

Le travail présenté ici constitue une étude préliminaire à l'obtention de monocristaux de $\mathrm{BaTiO}_{3}$ de structure quadratique par fusion du matériau. En particulier la substitution de faibles quantités de Baryum par le Strontium ou un Lanthanide semble une bonne alternative. Une étude spectroscopique de l'ion $\mathrm{Eu}^{3+}$ utilisé comme sonde structurale luminescente permet de suivre sans ambiguïté les transitions de phases ; l'étude approfondie de la répartition des ions $\mathrm{Eu}^{3+}$ dans la structure $\mathrm{BaTiO}_{3}$ est en cours.

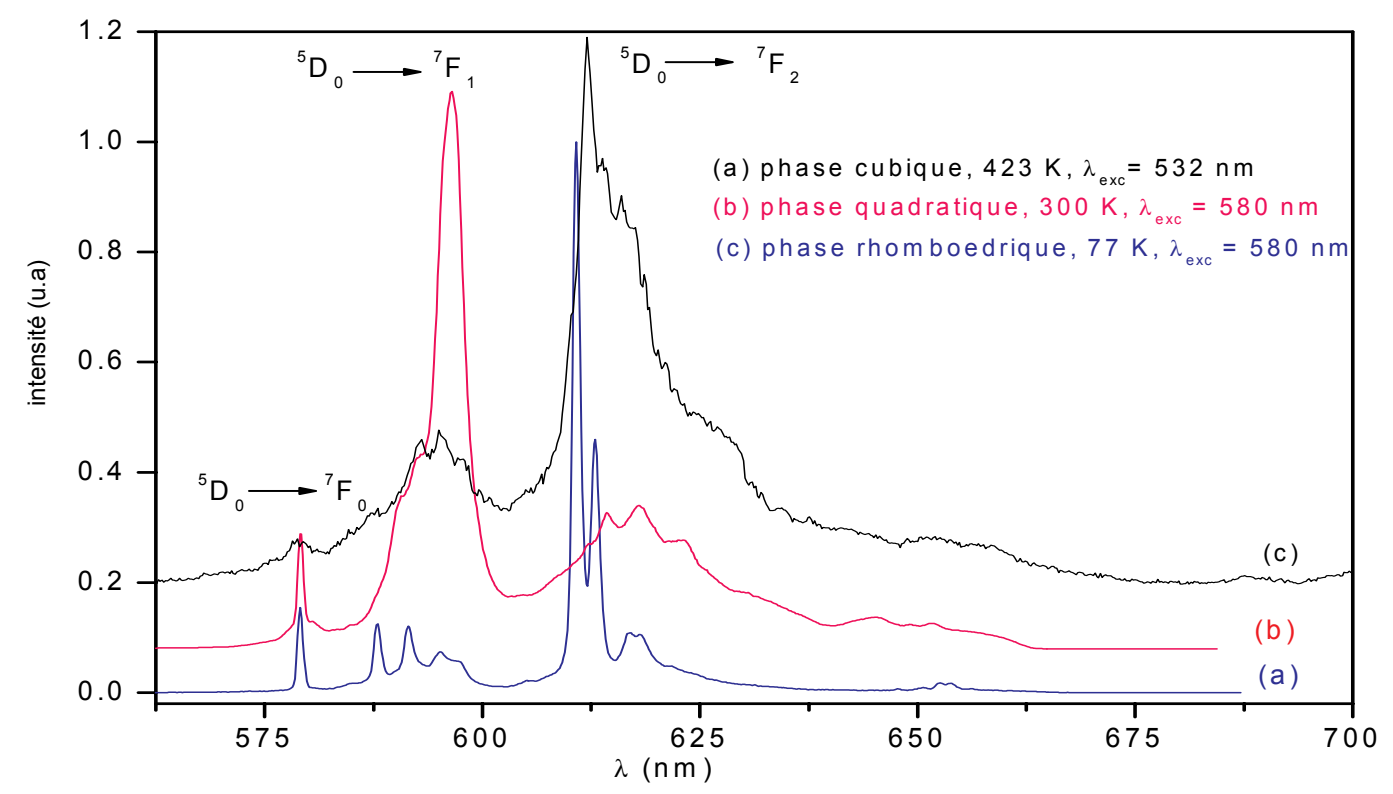

Figure 5. Spectre d'émission de l'ion $\mathrm{Eu}^{3+}$ dans $\mathrm{BaTiO}_{3}$ (a) fibre de structure cubique, (b) fibre de structure quadratique, (c) nanocristal de structure rhomboédrique.

\section{Remerciements}

Les auteurs remercient B. Bonnetot (LMI, Université C. Bernard-Lyon I) de son soutien. 


\section{Références}

[1] D. E. Rase, R. Roy, J. Am. Ceram. Soc., 38 (1955) 102.

[2] R. L. Townsend, J. T. La Macchia, J. App. Phys., 41 (1970) 5188.

[3] J. Feinberg, R. W. Hellwarth, Opt. Lett., 5 (1980) 519.

[4] J. Feinberg, Opt. Lett., 7 (1982) 486

[5] J. A. Basmajian, R. C. De Vries, J. Am. Ceram. Soc., 40 (1957) 373.

[6] B. A. Wechler, K. W. Kirby, J. Am. Ceram. Soc., 75 (1992) 981.

[7] H. Kojima, M. Watanabe, I. Tanaka, J. Cryst. Growth, 155 (1995) 70.

[8] J. Furukawa, T. Tsukamoto, Jap. J. App. Phys., 30/9B (1991) 2391.

[9] W. Strek, D. Hreniak, G. Boulon, Y. Guyot, R. Razik, Opt. Mat., 23 (2003) 15.

[10] H.E.Kay, P.Vousde, Philos. Mag., 40 (1949) 1019. 